\title{
Manifattura in transizione
}

\author{
ENZO RULLANI
}

\section{Why manufacturing matters?}

Sono passati quasi trenta anni da quando Cohen e Zysman (1987) titolavano: manufacturing matters. Ossia, la manifattura conta, è importante, per l'economia di un paese sviluppato. Era un grido (di dolore) che negli Stati Uniti si levava contro il "mito dell'economia post-industriale", come recita il sottotitolo del loro libro. E che reagiva alla perdita costante di peso, nel valore aggiunto e nell'occupazione, che la manifattura americana registrava da anni nei confronti dell'economia dei servizi. Un'economia che sembrava destinata non solo ad espandersi senza limiti, ai danni della manifattura, ma che aveva anche un effetto paralizzante, perché confinava in un ambito ristretto (la manifattura) le innovazioni tecnologiche e la crescita della produttività.

La manifattura conta, dicono Cohen e Zysman (e con loro molti economisti industriali), perché l'alternativa è l'asfissia di una economia dei servizi che non genera produttività e non alimenta l'innovazione.

Quello che è successo da allora non ha confermato questa profezia, ma non ha nemmeno fornito una risposta alternativa chiara e incontrovertibile. Piuttosto, l'evoluzione del nostro sistema produttivo ha cominciato a seguire percorsi complessi, perché sono entrati in gioco molti nuovi fattori: alcune innovazioni tecnologiche fondamentali, nel campo ICT ma non solo; la globalizzazione dei mercati e delle filiere produttive; la crescita di consumatori intelligenti e attivi, che sostituiscono gradualmente i consumatori di massa, condannati per principio dalle imprese della "vecchia" manifattura ad una condizione (fedele) di passività non intelligente.

Dalla crisi del fordismo in poi, il vento cambia: e sia la "vecchia" manifattura che i "vecchi" servizi perdono la loro compattezza, man mano che le imprese, nei due campi, mettono a punto nuove rotte e nuove vele per sfruttare la forza del nuovo vento, che si trovano di fronte.

Se oggi ci poniamo nuovamente la domanda di Cohen e Zysman di trenta anni fa, è perché molti interrogativi rimangono aperti. Bisogna infatti capire quello che è successo in questi trenta anni, per apprezzare il senso da dare ad affermazioni che

\footnotetext{
Professore di Economia della Conoscenza presso il TeDIS Center - Venice International University

e-mail: enzo.rullani@gmail.com
}

sinergie, rivista di studi e ricerche

n. 93, Gennaio-Aprile 2014, pp. 141-152

ISSN 0393-5108 - DOI 10.7433/ s93.2014.09 
oggi richiamano quel manufacturing matters che, pur essendo fondato su ottime ragioni, è stato dribblato, per così dire, dalla storia reale del capitalismo moderno.

Che tipo di ragionevoli obiettivi possono ad esempio avere politiche (europee) che, per i prossimi anni, puntano a mantenere la manifattura al $20 \%$ del PIL, con argomentazioni non troppo dissimili da quelle avanzate da Cohen e Zysman in passato?

E, per venire in Italia, che tipo di manifattura possiamo riprometterci di mantenere nei nostri distretti industriali, in presenza di sviluppi che tendono a riposizionare i nostri distretti nelle filiere globali, cambiando il peso e il senso della manifattura che può rimanere da noi, pagando costi assai maggiori di quelli richiesti altrove (Guelpa e Micelli, 2007)?

Diversi fattori, intervenuti negli ultimi trenta anni, hanno finito per mettere fuori gioco l'alternativa secca tra manifattura e servizi, come si poneva negli anni ottanta.

Prima di tutto, a partire dagli anni novanta, gli Stati Uniti diventano l'epicentro della crescita esponenziale dell'"economia digitale". Questa economia, basata sull'immaterialità del software, prima che sulla materialità dell'hardware, come era la precedente produzione di computer, rimette le imprese americane sulla frontiera di una evoluzione che, appena un decennio prima, sembrava destinata ad essere guidata dalla "nuova manifattura" flessibile costruita, in Giappone, intorno alla lean production e al toyotismo. $\mathrm{O}$, in Italia, intorno all'impresa diffusa e ai distretti industriali.

Si capisce, negli anni novanta, che la sfida del futuro, tra i paesi sviluppati, non sarà tra nuova e vecchia manifattura, ma tra l'economia digitale (chiamata significativamente new economy), che è altamente smaterializzata, ed effervescente, e l'economia "pesante" della produzione di beni materiali, dall'altra.

L'economia digitale crea valore utilizzando bit informatici che transitano a valanga su Internet, dando un mondo interconnesso di relazioni, significati, emozioni e aspettative sul futuro possibile, che la borsa registra immediatamente, anche se forse con troppo precoce entusiasmo.

In parallelo, la manifattura tradizionale (di fabbrica), sul finire del secolo, comincia a sentire il peso di tutta una serie di fardelli che ne ostacolano il "volo", nonostante le innovazioni che possono renderla flessibile: l'insostenibilità ambientale per la dissipazione energetica e gli scarti; la crescente insofferenza del lavoro per i metodi fordisti e autoritari di organizzazione della fabbrica; la ricerca di significati e identità nuove, da parte di consumatori che sono ormai saturi di beni materiali di prima necessità.

Dunque la prospettiva che se ne ricava, negli ultimi anni del novecento, è che il secolo del fordismo finisce col declino della fabbrica (materiale), sostituita, nella generazione del valore, dalla rete delle tante invenzioni e produzioni immateriali, che si incontrano su Internet. 


\section{L'economia-mondo, dopo il 2000}

Poi, finisce il secolo, e la prospettiva cambia di nuovo. Il boom della new economy implode in uno sboom che ne riduce le pretese e le aspettative. L'economia digitale continua anche dopo il 2000 sottotraccia a cambiare il mondo, che si troverà di lì a poco iper-connesso mediante smartphones, tablets, social networks dotati di grande potere di penetrazione e di condizionamento. Ma il suo fisiologico rallentamento non rimette affatto in pista la "manifattura che conta". Anzi.

Accade qualcosa che non ci si aspettava. Le nuove tecnologie della comunicazione (ICT, Internet) e la globalizzazione dei mercati hanno reso facilmente riproducibili e facilmente trasferibili le conoscenze codificate che, nei paesi sviluppati, sono state elaborate sotto forma di sapere scientifico, di tecnologie incorporate nelle macchine, di procedure standard di lavorazione che le aziende specialmente le grandi aziende - hanno reso impersonali e astratte, ossia indipendenti dalle persone e dal contesto di uso (Rullani, 2014).

La fabbricazione di oggetti materiali è in genere una attività altamente codificata, dove ogni singola operazione è dettata da un programma che va eseguito, possibilmente senza varianti rispetto alla norma. Sebbene ci sia comunque una parte (residuale) di abilità pratica che viene richiesta agli operai e ai quadri, nella fabbricazione l'intelligenza generativa è concentrata ai vertici delle aziende, quando le nuove idee vengono tradotte in modelli replicabili che poi sono "industrializzati" da programmi a cui lavorano ricercatori, progettisti, programmatori e manager di varia competenza. Per il resto, la catena produttiva richiede intelligenza replicativa (che esegue il modello dato) e non generativa.

Questa intelligenza è impersonale, nel senso che - rispondendo ad un codice astratto - può essere messa al lavoro, con opportuni programmi e investimenti in capacità di assorbimento, anche nei tanti paesi low cost che si affacciano all'industrializzazione. Di qui un imponente flusso di attività manifatturiere che si spostano, anno dopo anno, verso i paesi emergenti. Lasciando ai paesi sviluppati attività che puntano sulla conoscenza generativa (anche immateriale), prima che sulla manifattura replicativa di fabbrica.

Dal 2000 in poi, abbiamo assistito ad un imponente flusso di conoscenza codificata - in gran parte manifatturiera - che è stata attratta da localizzazioni low cost, consentendo alle multinazionali che gestiscono questa trasformazione di "catturare" il surplus di valore corrispondente.

Con effetti dirompenti. La Cina, nel 2000 aveva 1'8\% della produzione manifatturiera mondiale. Undici anni dopo, nel 2011, è arrivata al 22\% (dati Global Insight). Contemporaneamente gli Stati Uniti sono scesi dal 24,8 al $14,5 \%$, e il Giappone - la patria della "nuova manifattura" degli anni ottanta - dal 15,8\% al 9,4\%. Tutti i paesi europei perdono quota, compresa la Germania (dal 6,6 al 6,3\%), anche se in maniera diseguale. L'Italia che aveva nel 2000 una quota pari al 4,1\% regge bene fino al 2007, ma poi scivola al 3,3\% per effetto della crisi, e della caduta verticale della domanda interna. 
In tutti i paesi dunque, si comincia a distinguere tra la manifattura replicativa, standard, destinata ad essere attratta dai paesi low cost, e manifattura innovativa, che invece ha buone possibilità non solo di restare, ma anche di catturare quote importanti di surplus dalle filiere mondiali a cui partecipa. Il cuore della manifattura innovativa che dà spazio all'intelligenza generativa delle persone e del contesto locale non è la fabbrica, ma un circuito di attività immateriali come l'ideazione, la ricerca e sviluppo, il design, l'innovazione, la modellizzazione e programmazione della produzione, la logistica, la comunicazione, la gestione degli ordini nelle filiere globali, i marchi e i significati connessi, la commercializzazione, il rapporto sempre più interattivo col mondo della distribuzione e del consumo. La fabbrica può completare questo ciclo di attività immateriali in tutti i casi in cui la produzione non è codificata e programmata in astratto, ma richiede invenzioni, adattamenti, flessibilità, contributi da parte di persone in carne ed ossa, che governano le macchine in funzione della loro intelligenza generativa. Accade tutte le volte che la fabbricazione si allontana dallo standard, facendo posto a soluzioni flessibili o personalizzate, a piccole serie o prodotti unici, a modelli sperimentali e campionari che vengono messi a punto in laboratori in cui prevale lo spirito dell'artigianato di qualità, piuttosto che quello della produzione di massa.

Accanto alla scoperta della "manifattura di qualità", o meglio della manifattura intelligente sopra richiamata, il nostro tempo è dominato dalla riscoperta dei servizi, e dunque della funzione sempre più rilevante che essi hanno nei processi di innovazione.

La maggior parte delle attività generative che formano il "terziario interno" della singola impresa corrispondono ad un "terziario esterno" fatto di aziende di servizi e di professionisti che vengono coinvolti nelle attività di innovazione e di immaginazione del futuro possibile, o che forniscono servizi ad alto valore aggiunto necessari alla trasformazione in corso (Rullani, 2013). Si pensi alla comunicazione dei marchi e dei significati; al reperimento di mezzi finanziari; alla formazione del capitale umano; alla creazione di sistemi logistici duttili ed efficienti che collegano il locale col globale; al presidio delle nuove tecnologie e dei nuovi mercati; alla gestione di reti ICT sempre più complesse e pervasive; alla fornitura di conoscenze e servizi personalizzati che aiutano i clienti ad affrontare le situazioni nuove con cui hanno quotidianamente a che fare.

Anche il terziario, dunque, ormai si trova stretto nell'abito che gli era stato ritagliato in epoca fordista e poi negli anni ottanta. Al suo interno si sta infatti sviluppando un nucleo sempre più rilevante di attività che non ha niente a che fare con la vecchia nozione di terziario improduttivo o inerziale, ma che è invece un propulsore essenziale dell'innovazione, alla pari del terziario interno alle aziende.

\section{II background ereditato dal novecento: la terziarizzazione implosiva}

La riscoperta dei servizi in chiave innovativa cambia radicalmente la contrapposizione tradizionale che, in passato, ha visto nei servizi e nella manifattura 
due polarità opposte, anche se complementari. In particolare, se i servizi possono essere parte del flusso di innovazioni che caratterizza il nostro presente, viene a cadere l'idea di un rapporto tra manifattura e terziario che si gioca nel quadro del cosiddetto Baumol desease (la malattia da costi). Un'idea che ha caratterizzato il fordismo maturo (Baumol, 1985), e che per un certo tempo ha consegnato tutto il mondo dei servizi ad un ruolo parassitario e regressivo.

Negli anni ottanta, gli Stati Uniti soffrono ormai da tempo di una stagnazione della produttività, che Baumol e altri studiosi attribuiscono al peso sempre più grande del terziario rispetto al settore industriale. È un trend leggibile, fino agli anni novanta, un po' in tutti i paesi industrializzati, ma soprattutto in quelli, come gli Stati Uniti, che hanno un'industria matura.

La ragione di questo spostamento dell'occupazione e del valore aggiunto verso il settore dei servizi, a scapito della quota della manifattura deriva, paradossalmente dal fatto che la manifattura - lavorando a stretto contatto con la tecnologia e dovendo servire un mercato molto competitivo - fa bene il suo mestiere, aumentando anno dopo anno la produttività, ossia il numero dei "pezzi" in uscita dalle linee produttive per ogni ora lavorata.

$\mathrm{Ma}$, se la domanda dei prodotti materiali così ottenuti non cresce allo stesso ritmo - e non cresce perché ormai molti di quei prodotti sono già presenti in abbondanza nelle case e nei garage dei consumatori americani - l'aumento continuo della produttività industriale si traduce in una corrispondente riduzione dell'occupazione richiesta nelle fabbriche e in una flessione dei prezzi dei prodotti industriali, che scendono per effetto della diminuzione dei costi.

Che cosa fanno i consumatori con i soldi che risparmiano dai minori acquisti di prodotti materiali e dalla decrescita continua del loro prezzo?

Se tenessero questi risparmi inoccupati, in banca o sotto il materasso, avremmo una depressione da caduta della domanda. Ma, per fortuna non è così: i consumatori scoprono nuovi bisogni e la maggior parte di questi alimenta la domanda di servizi: la gente va al cinema, alle partite, in palestra, al ristorante, in gita turistica. $\mathrm{O}$ si innamora dei prodotti di lusso (abiti, profumi, borse, gioielli ecc.) che vengono sì forniti dalla manifattura ma che richiedono un forte investimento nei servizi che li accompagnano, in particolare nella creazione di significati, nella comunicazione dei marchi e nella rete commerciale diretta (monomarca) rivolta al consumatore finale. Oppure, ancora, i consumatori personalizzano di molto le loro esigenze e questo alimenta la crescita dei cosiddetti global services, ossia di aziende terziarie che si prendono cura della casa, del giardino, della manutenzione e riparazione delle case, e così via.

Non è solo il consumatore finale che alimenta la terziarizzazione dell'economia. Anche la Pubblica Amministrazione, col peso crescente del welfare, drena risorse dall'economia privata (attraverso le tasse) e le restituisce sotto forma di servizi, che hanno un peso crescente sull'occupazione e sul PIL complessivo.

D'altra parte, le stesse imprese industriali, per aumentare la produttività della fabbrica ricorrono in modo crescente a servizi manageriali, organizzativi, informatici, manageriali, creativi (design, stilisti, ricerca e sviluppo), formativi e di 
gestione del lavoro, che si aggiungono ad un impegno sempre più forte sul piano della commercializzazione. Questi servizi in parte sono "interni" all'azienda manifatturiere, ma in parte ricorrono al terziario "esterno" diventando un veicolo visibile della terziarizzazione del sistema complessivo (Bettiol et al., 2012).

Il fordismo, nella sua fase di maturità, diventa in questo modo una service economy, in cui gran parte degli occupati e del valore aggiunto (con percentuali che vanno dal 60 all' $80 \%$, compresa la pubblica amministrazione) sono dovuti al settore terziario (Rullani, 2012).

Non si tratta di una semplice trasformazione dei pesi tra un settore e un altro. Ma di una terziarizzazione implosiva che - secondo la visione pessimistica della "malattia da costi" - paràssita la crescita industriale con la crescita continua dei servizi e dei loro costi, fino a soffocare lo sviluppo tout court.

Protagonisti della terziarizzazione implosiva sono i due settori - manifattura e servizi - visti, fino agli anni novanta, come settori antitetici, radicalmente diversi. Con uno slogan potremmo dire che l'immagine prevalente alimentata dal Baumol desease era quella di una manifattura che genera produttività e di un terziario che la "consuma". Nel senso che il valore ricavato dalla manifattura viene speso per tutta una serie di servizi ciascuno dei quali è utile, ma refrattario all'innovazione e agli aumenti di produttività. Non siamo all'idea degli economisti classici per cui i servizi erano "improduttivi" tout court, ma poco ci manca.

\section{Tra materiale e immateriale}

Che cosa impediva ai servizi di seguire la manifattura lungo la strada dell'innovazione e degli incrementi di produttività?

Allora la risposta sembrava semplice e convincente: la natura immateriale del servizio, che - nel momento in cui la prestazione utile viene fornita al cliente impedisce di separare domanda e offerta. Ciascuna prestazione "lega" l'offerta alla domanda, perché va fornita nel momento e nel luogo in cui si trova la domanda: la singola domanda, del cliente che domanda un pasto al ristorante o una lezione al formatore. Le possibilità di aggregare la domanda, per fornire un'offerta congiunta, collettiva, sono limitate: il ristoratore potrà mettere nel suo locale dieci o venti tavoli, ma poi dovrà servire ai clienti quello che ciascuno di loro sceglie dal menu ${ }^{1}$.

1 Solo in casi eccezionali (porti, aeroporti, stazioni ferroviarie, uffici della P.A., scuole, ospedali) esiste un potere dell'offerta che riesce a concentrare la domanda (nel tempo e nello spazio) facendola confluire nel programma operativo stabilito dall'offerta. In parte, le capacità di concentrazione della domanda sono anche quelle che rendono possibile lo sviluppo in forme moderne (e meccanizzate) della GDO (grande distribuzione organizzata), a cui i consumatori arrivano con la propria auto in centri commerciali situati fuori città. In questo modo l'offerta di servizi può fare delle economie di scala, standardizzando il servizio con una metodologia para-industriale, anche se spesso questo modo di produrre il servizio va a scapito della flessibilità e della personalizzazione a cui il sistema tradizionale dei servizi ci ha abituato. 
L'impresa manifatturiera invece ha una chance in più, perché essa può separare i propri flussi produttivi dalla domanda dispersa e fluttuante che esiste nell'ambiente, usando il prodotto materiale, che è stoccabile nel tempo e trasferibile nello spazio, come fattore disgiuntivo, che - grazie alla logistica dei magazzini e dei trasporti rende reciprocamente indipendenti domanda e offerta, sia per quanto riguarda il tempo che lo spazio (Di Bernardo, 1992).

Nei primi due secoli della modernità (dal 1800 fino al 2000), questo grado di libertà, messo a disposizione dal prodotto materiale, è stato essenziale per l'offerta che ha meccanizzato il processo produttivo e fatto economie di scala (in termini di volumi) proprio contando sulla possibilità di concentrare la produzione in una grande fabbrica, e dunque in un luogo puntuale, da cui servire una domanda che resta magari dispersa in un territorio molto esteso. Lo stesso vale per il tempo: la fabbrica industriale poteva funzionare in base al suo programma, e raccordarsi con la domanda (fluttuante) usando come buffer il magazzino in cui confluiscono i prodotti finiti, in attesa di essere venduti.

I servizi non potevano farlo.

Ecco la radice della malattia da costi che, nel fordismo maturo, colpisce prima $\mathrm{i}$ servizi e poi l'intera economia: la produzione dell'immateriale (ossia del servizio), non avendo alcun elemento disgiuntivo efficace, è tagliata fuori dalla modernizzazione, che richiede la concentrazione dell'offerta in una "fabbrica" e la programmazione autonoma dei ritmi prodottivi, rispetto alla domanda.

Ma questa condizione dell'immateriale non modernizzabile cessa di esistere quando, a partire dagli anni novanta, abbiamo il progressivo sviluppo delle reti digitali che, in una certa misura, invertono i ruoli: l'immateriale (tradotto in bit) diventa trasferibile in tempo reale e a costo zero da una parte all'altra del mondo, mentre il materiale è limitato - in questo - da costi di trasporto e di magazzinaggio che non sono indifferenti.

Le reti digitali hanno dunque l'effetto di "liberare" l'immateriale e i servizi dalle catene che, in precedenza, impedivano loro di accedere alle forme moderne di produzione e consumo. Non è certo un caso se tutte le aziende di maggiore successo degli ultimi anni nascono nel campo dell'economia digitale, e dell'immateriale (Rullani, 2012).

Non tutti i servizi, certo, sono traducibili in un flusso di bit che può rispondere ad una domanda collocata a cento o mille chilometri di distanza. Un servizio di pulizia delle strade, o delle stanze, richiede di essere svolto qui-e-ora, senza grandi gradi di libertà per l'offerta.

Ma la disgiunzione tra domande e offerta può essere praticata senza grandi difficoltà per tutti quei servizi che forniscono alla domanda conoscenze codificate, una volta che queste sono state tradotte in un software o in un data base numerico. ̀े la strada che porta alla proliferazione dei call center, o dei bancomat, in sostituzione degli sportelli rivolti al pubblico. O è quella che sposta nella cloud (nuvola) i data base delle grandi organizzazioni, rendendoli così accessibili da molte diverse posizioni. L'e-commerce sostituisce gradualmente le transazioni meno complesse, e più codificabili. Macchine, fabbriche e residenze possono sempre di più essere 
controllate e guidate a distanza, considerando che ormai anche le operazioni chirurgiche possono essere fatte con il paziente che sta a Milano mentre il chirurgo che lo opera guida gli apparecchi e i robot necessari dalla sua clinica di New York (Di Maria et al., 2012).

Non sono solo le prestazioni basate sulla conoscenza codificata ad essere liberate dalla barriera della distanza. La disgiunzione spazio-temporale tra chi offre e chi domanda un servizio è anche possibile (ma non a costo zero) per quelle prestazioni in cui devono essere impiegate conoscenze generative che richiedono l'intervento della mente umana per interpretare, immaginare, convincere, prendere decisioni in condizioni di incertezza. In questo caso la relazione a distanza (nello spazio e nel tempo) non è a costo zero e in tempo reale, ma richiede la pazienza di una comunicazione interpersonale che, tuttavia, può ormai avvenire in forme efficaci anche attraverso Skype, le videconferenze, i collegamenti in mobilità, il co-working fuori dai luoghi canonici della fabbrica e dell'ufficio.

In una serie crescente di casi, l'immateriale consente dunque all'offerta del servizio di auto-organizzarsi nelle forme più convenienti usando le ICT per raccordarsi con la domanda. Ma in questo modo la barriera che escludeva i servizi dai processi di modernizzazione, e dalle loro economie di scala, viene meno.

$\mathrm{Nel}$ momento in cui salta la demarcazione storica tra materiale (manifatturiero) e immateriale (servizi), la partita ricomincia, con un nuovo giro di carte.

Il primo effetto di questo cambiamento è che una parte importante terziario (immateriale) oggi può essere "industrializzato", come e più del manifatturiero (materiale), usando tutta la potenza delle tecnologie replicative della modernità. Milioni di persone, tutti i giorni, usano motori di ricerca come Google o frequentano luoghi dedicati (virtuali) nei social networks: i volumi dei servizi offerti e usati sono così elevati che molti di questi sono gratuiti, essendo sufficiente il guadagno pubblicitario a finanziare il servizio reso. Ma anche quando paghiamo qualche euro per una delle tante apps che personalizzano il servizio standard, partecipiamo ad un processo che ha alti volumi e bassi costi di replicazione.

Accanto a questo processo di industrializzazione dei servizi codificati o comunicabili a distanza, assistiamo ad un cambiamento di ruolo della manifattura. Che ormai, su questa scia, ricava solo una parte del valore dalla vendita del prodotto materiale, mentre punta sempre di più sulla capacità di creare e vendere significati o esperienze emotivamente coinvolgenti associate al prodotto materiale. L'industria, in altri termini, si sta smaterializzando. E diventa per certi versi simile, per problemi e modalità di azione al mondo dei servizi. I quali, muovendosi in modo convergente, stanno a loro volta esplorando i vantaggi di modelli di business vicini alla tradizione industriale (economie di scala, modularità, standard ecc.).

La frontiera dell'innovazione vede oggi affiancate l'industria che investe sull'immateriale e i servizi che si industrializzano, cercando di fare efficienza ed economie di scala. La convergenza supera vecchie dicotomie e richiede nuove distinzioni.

La globalizzazione poi, negli ultimi anni, ci ha messo del suo, perché ha reso conveniente a molte multinazionali il decentramento di alcune fasi della filiera 
produttive (in genere quelle riguardanti attività replicative e conoscenze codificate) nei paesi emergenti. Ma per conseguire i vantaggi della globalizzazione, anche in termini di sfruttamento delle differenze di costo e di capacità tra i vari paesi del mondo, bisogna rendere trasferibili e replicabili molte delle conoscenze impiegate nella manifattura e nei servizi connessi, da trasferire.

In passato, la fabbricazione dei prodotti utilizzava anche l'abilità personale degli operai impiegati nelle lavorazioni e le risorse connettive esistenti di uno specifico contesto (la fabbrica, l'ambiente in cui essa si situa). Processi del genere, tuttavia, sono difficilmente trasferibili altrove, in luoghi in cui quelle abilità e quelle risorse connettive non ci sono o sono diverse dal modello di origine. Per trasferire fasi o funzioni produttive in un paese low cost, bisognerà dunque - prima - rendere codificato e impersonale il lavoro degli uomini, separando la loro intelligenza personale dai processi di trasformazione materiale in cui sono coinvolti.

La codificazione che spersonalizza i processi materiali, astraendo dalle persone e dal contesto, è un problema serio per le grandi imprese che sono impegnate in questa trasformazione dei loro modelli di business. Ma è un problema drammatico se si pone in un distretto industriale come quelli che sono oggi l'ossatura del nostro capitalismo industriale. Nel distretto, tipicamente, i processi produttivi sono rimasti da sempre strettamente legati alla materialità delle persone e del contesto culturale del distretto. Trasferire fasi e funzioni della filiera dal distretto ad altro luogo, come accade sempre più spesso sotto la pressione della concorrenza internazionale implica una rottura del legame tra i processi e le persone, tra i luoghi della produzione e il contesto culturale e sociale in cui essa si svolge.

In prospettiva, l'aumento delle distanze tra fasi e funzioni svolte nelle nuove filiere globali, implica una crescente smaterializzazione dei processi produttivi, nel senso che questi vengono codificati in forma astratta, separandoli dalle persone e dai contesti di origine, in modo da renderli modulari e trasferibili altrove.

Così facendo, la conoscenza associata alle operazioni di trasformazione materiale e di uso del prodotto viene codificata, diventando trasferibile anche a prescindere dal supporto materiale (la macchina, il prodotto, il componente) in cui era in origine incorporata. Questo spinge, ogni volta che si può, a trasferire non più l'oggetto materiale, ma la conoscenza che serve per produrlo: costa meno e arriva più rapidamente. La manifattura si sta in altri termini spostando a valle nelle filiere, mentre il grande campo dell'economia globale è sempre più dominato dai flussi di conoscenza che collegano le funzioni a monte con quelle a valle della filiera.

Oggi ci si domanda, ad esempio, se conviene produrre elettrodomestici, qui per venderli in giro per il mondo, o se conviene, invece, codificare le conoscenze necessarie, venderle o trasferirle in forma immateriale, costruendo le fabbriche vicino ai mercati di sbocco, in modo che possano lavorare on demand, vicine ai luoghi del consumo. Questa unità manifatturiere "a valle", che possono lavorare in stretta interazione con gli users industriali o finali, ricevono le conoscenze che servono da detentori che stanno a distanza, e che forniscono il "servizio" del loro sapere a tutti gli users del mondo che lo richiedono. 
È sotto gli occhi di tutti il fenomeno dei makers, che usano le conoscenze (codificate) disponibili o acquistabili in rete, per auto-produrre a casa oggetti complessi, che una volta sarebbero stati delegati senz'altro al lavoro di una fabbrica manifatturiera lontana e standardizzata (Anderson, 2012). Le stampanti 3D sono un'ulteriore tappa di questo cammino che porta sempre più vicino al consumo (in piccole fabbriche neo-artigianali o direttamente presso il consumatoreautoproduttore) le fasi della manifattura in cui si tratta di personalizzare il prodotto e inventare varianti dotate di significato per chi lo usa. Ma lo stesso potrà accadere, e forse sta accadendo, per i libri e l'editoria: le conoscenze codificate viaggiano in rete in forma immateriale, e si ri-materializzano solo nel momento in cui si arriva al consumo finale (stampando il libro in casa o in una stamperia sotto casa, o riproducendo sulla carta gli articoli che di volta in volta siamo interessati a leggere).

In questo modo acquistano nuovi spazi di lavoro quei neo-artigiani che, partendo dal rapporto diretto con il consumatore finale, usano la loro creatività per inventare oggetti o stili di vita ricchi di significato e di qualità per chi li dovrà utilizzare (e pagare). In Italia abbiamo una buona base di partenza per questi sviluppi, un po' in tutti i settori del Made in Italy (Micelli, 2011).

Intendiamoci: non è una tendenza che potrà investire tutta la manifattura. Sicuramente le acciaierie e le grandi centrali elettriche rimarranno a monte, nelle filiere, ben separate dalla domanda finale, ma i vantaggi della smaterializzazione delle conoscenze che le rende moltiplicabili e trasferibili sono tali da spostare progressivamente il baricentro intellettuale e creativo delle filiere verso le fasi terminali, a valle, vicino al consumo finale. Dando vita a trasformazioni materiali che, certo usano componenti e materiali prodotti a monte, ma che sempre di più assemblano i moduli cognitivi e materiali del puzzle vicino all'user, e alla sua intelligenza.

Dunque, ormai, c'è manifattura e manifattura; e, in parallelo, possiamo dire, c'è terziario e terziario. Non solo la vecchia demarcazione è venuta meno, ma sono cambiati i significati dei termini. Per parlare del futuro bisogna specificare: con quale manifattura abbiamo a che fare? E con quali servizi?

\section{Che ruolo ha la manifattura nel nuovo paradigma, post-2000?}

Questi mutamenti di fondo (ICT, mass media, globalizzazione) cambiano alla radice la demarcazione tradizionale tra industria e terziario. $\mathrm{Ne}$ deriva, accanto all'industria di massa che continua ad essere tale e diventa industria di commodities globali a basso costo, una neo-industria che invece ricerca la qualità e dunque comincia ad offrire al cliente personalizzazione, varietà, significati, esperienze e garanzie che una volta erano tipiche dei servizi.

Similmente, in senso opposto ma convergente, una parte dei servizi si trasforma in neo-servizi, ossia in attività che - senza passare per un prodotto materiale - si industrializzano nella produzione e - pur garantendo un certo grado di flessibilità - 
forniscono agli users servizi standard con costi di riproduzione e di trasferimento praticamente nulli.

In questa nuova divisione del lavoro anche una parte dell'artigianato, che in precedenza era industria dai piccoli numeri, cambia natura e diventa artigianato di qualità, che vende insieme la qualità del lavoro personalizzato e i significati/marchi di larga scala che lo caratterizzano rispetto ai prodotti industriali standard (Micelli, 2011).

Il risultato è che la distinzione tradizionale tra settori (manifattura, terziario ecc.) viene meno e oggi sarebbe difficile dire se un'azienda come la Geox è un'azienda manifatturiera o un'azienda integralmente terziaria. Lo stesso vale per un'azienda tipo Google, che vende un servizio standard caratterizzato dai grandissimi volumi, e lo vende a scala mondiale, anche se il prodotto è un "servizio", non un bene.

Ciò che conta è l'intelligenza che viene messa in campo dalle imprese, sia nella produzione dei beni materiali che dei servizi o beni immateriali. Industria e servizi intelligenti, nelle filiere globali, possono rimanere nei territori ad alto reddito/costo perché fanno valere la loro intelligenza differenziale e non trasferibile rispetto ad altre localizzazioni low cost.

Insomma, abbiamo bisogno di ridefinire le variabili che caratterizzano le attività, superando i settori tradizionali e guardando al loro contenuto di intelligenza, non al loro contenuto in termini di materiale/immateriale.

Come?

Serve una riflessione collettiva che situi il pensiero e la pratica manageriale in questa transizione verso un futuro che, per adesso incombe minaccioso su di noi, ma che invece va compreso nelle sue potenzialità e costruito in base ad un progetto consapevole (Rullani, 2011).

\section{Bibliografia}

ANDERSON C. (2013), Makers. Il ritorno dei produttori. Per una nuova rivoluzione industriale, Rizzoli, Etas, trad. italiana (2012) di Makers. The new industrial revolution, Random House, New York.

BAUMOL W.J. (1985), "Productivity policy and the service sector", in Inman R.P. (a cura di), Managing the Service Economy: Prospects and Problems, Cambridge University Press, Londra, cap. 11, pp. 301-337.

BETTIOL M., DI MARIA E., GRANDINETTI R. (2012), “Codification and creativity: knowledge management strategies in KIBS”, Journal of Knowledge Management, vol. 16, n. 4, pp. 550-562.

COHEN S.S., ZYSMAN J. (1987), Manufacturing Matters. The Mith of the Post-Industrial Economy, Basic Books, New York.

DI BERNARDO B. (1992), "La produzione neo-industriale e i servizi”, Sinergie, n. 29, pp. 115-136.

DI MARIA E., GRANDINETTI R., DI BERNARDO B. (Eds.) (2012), Exploring Knowledge-Intensive Business Services. Knowledge Management Strategies, Palgrave, Cheltenham. 
MICELLI S. (2011), Futuro artigiano. L’innovazione nelle mani degli italiani, Marsilio, Venezia.

GUELPA F., MICELLI S. (2007), I distretti industriali del terzo millennio. Dalle economie di agglomerazione alle strategie di impresa, Il Mulino, Bologna.

RULLANI E. (2011), "Verso una società imprenditoriale consapevole", in Nardozzi G., Paolazzi L. (a cura di), Costruire il futuro. PMI protagoniste: sfide e strategie, Sipi, Roma, pp. 39-102.

RULLANI E. (2012), "Produttività e servizi: una storia di divergenze parallele", in Rullani E., Cantù C., Paiola M., Prandstraller F., Sebastiani R., Innovazione e produttività. Alla ricerca di nuovi modelli di business per le imprese di servizi, Franco Angeli, Milano, pp. 35-96.

RULLANI E. (2013), “Un'altra idea di futuro”, Complessità e sostenibilità nel progetto, n. 5, gennaio-marzo, pp. 6-11.

RULLANI E. (2014), "Territori in transizione. Il nuovo rapporto tra imprese e politiche territoriali per la rinascita industriale e l'innovazione", in Cappellin R., Marelli E., Rullani E., Sterlacchini A. (a cura di), Crescita, investimenti e territorio. Il ruolo delle politiche industriali e regionali, Website "Scienze Regionali" (www.rivistasr.it), eBook. 